\title{
Safety Confirmation in Restroom using Ultrasonic Sensors for Elders in Outlying Island
}

\author{
Shenglin $\mathrm{Mu}^{\mathrm{a},{ }^{*}}$, Shota Nakashima ${ }^{\mathrm{b}}$, Kanya Tanaka ${ }^{\mathrm{b}}$ \\ aHiroshima National College of Maritime Technology, 4272-1, Higashino, Osakikamijima-cho, \\ Toyota-gun, Hiroshima 725-0231, Japan \\ ${ }^{\mathrm{b}}$ Graduate School of Science and Engineering, Yamaguchi University, 2-16-1, Tokiwadai, \\ Ube, Yamaguchi, 755-8611, Japan
}

*Corresponding Author: mshenglin@ @iroshima-cmt.ac.jp

\begin{abstract}
The aging problem in Japan goes more and more serious. For lightening the burden of care workers in Japan, systems to confirm safety of elders in daily life attract a lot of attentions in recent years. In this research, a safety confirmation system for elders living in outlying island is designed. As a part in the system, a motion detection system using ultrasonic sensor is reported for safety confirmation of elders living in outlying island in Japan in this paper. Ultrasonic sensors are employed as observers for object detection and distance measurement in restroom for safety confirmation. According to privacy protection requirement in nursing, the ultrasonic sensors apply no image or video information are good for applications in daily care of elders. By employing the proposed method, motion of target person can be detected. The effectiveness of the proposed method is confirmed by experiments.
\end{abstract}

Keywords: motion detection, safety confirmation, privacy protection, ultrasonic sensor.

\section{Introduction}

As the aging society problem goes heavier, the number and proportion of elders are becoming larger and larger in the population of Japan. Especially, in some outlying islands the situations are more serious. For example, the island in Hiroshima named as Osakikamijima, which is an outlying island with the population of 8,448 in 2010 , has the rate of aging around $40 \%$. Among the elders in the island, there are also almost 1,200 elders living along. The burdens of elders' nursing and daily care are heavy to their families and the government. It is not an easy work even just confirming their safety in daily life. Resources cost in nursing are supposed to increase more and more in near future. Therefore, in engineering fields, there are many researches for lighten the care burden proposed in recent years. Especially, for the safety confirmation, there are already some products introduced in real applications ${ }^{(1)}$.

Generally, the surveillance cameras are most widely applied in safety confirmation. However, there are some limitations in applications of cameras. First of all, the video or image signals fetched by cameras contain too much information more than we want for safety confirmation in daily life. Owing to privacy protection policy, they cannot be applied in private space, such as restroom, bath room and bed room and etc. The places are just where accidents happened a lot. On the other hand, the cost of camera systems are usually high, especially, some systems with the ability to record in dark environment ${ }^{(2)}$.

Therefore, sensors are more widely applied in safety confirmation systems in recent years. In this research, a safety confirmation system with sensors for elders living in outlying island is proposed. As a part of the proposed system, the application of ultrasonic sensor for the system is reported in this paper. The proposed method using ultrasonic sensor is simple and low cost. For evaluating the effectiveness, the sensor was applied in safety confirmation in restroom, where there are many falling accidents happened. And the experimental results are discussed in this paper. The paper is organized as follows. Section 1 is the general introduction. After this introduce section, the principle of ultrasonic sensor is introduced in Sect. 2. The experiments for effectiveness confirmation are introduced in Sect. 3. Section 4 gives 
conclusions.

\section{Ultrasonic Sensor}

\subsection{Principle of distance measurement}

Ultrasonic sensor is a kind of distance sensors, which employs ultrasonic sound for measuring distance without physical contact. The sensors are well applied in distance measurements and objects detecting without considering about the shape or appearances of objects. Figure 1 shows the basic principle of distance measurement. From the sending unit, an ultrasonic pulse is transmitted. The pulse is higher than $20 \mathrm{kHz}$, over the range of human hearing. The time of echo from object, received by the receiving unit, is employed in estimation of the distance to object. In this research, it is used for detecting the distance from sensor to target person. As Fig. 1 shows, if we define the distance between the object and sensor as $L$, it can be estimated by the following equation.

$$
\mathrm{L}=\mathrm{V} \times \frac{s}{2}
$$

where $V$ is the velocity of sound, and $s$ is the time cost from sending to receiving. We usually consider the velocity of sound in air as $340 \mathrm{~m} / \mathrm{s}$. Then, the distance can be estimated easily.

\subsection{Detection device}

For the aims of simplification and low cost, a module of Parallax's PING))) is employed in the detection device. Fig. 2 shows the photograph of the sensor. There are 3 pins in the module, which are signal, voltage and ground, respectively. The signal fetched by the sensor is processed by Arduino board of Sainsmart UNO, which is quite simple and popular in electronic applications recently. By employing the devices, it is easy to realize distance measurement in each second.

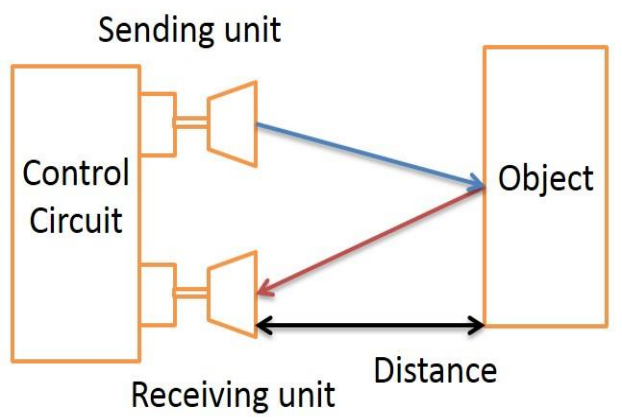

Fig. 1. Principle of ultrasonic sensor

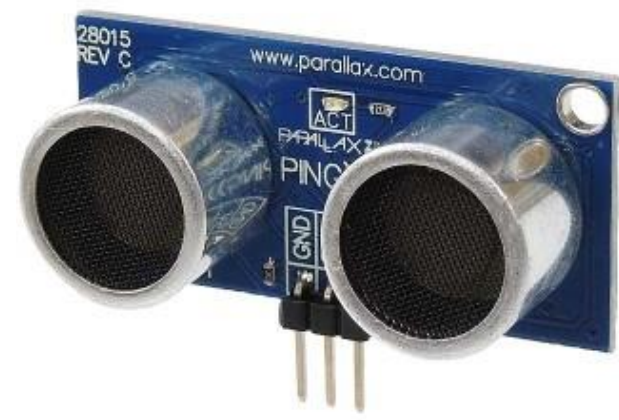

Fig. 2. Layout of experimental environment

\section{Experiments}

For evaluating the effectiveness of the proposed method, a group of experiments have been implemented.

\subsection{Experimental setting}

A simple experimental environment of restroom is employed in this research. In the restroom, surveillance cameras are not allowed to be applied. The ultrasonic sensors introduced above are designed for detecting the motion of target person correctly in this research. As we see in Fig. 3, it is the layout of restroom, which is applied in experiments. The sensors are set at the positions of 200 centimeters from the entrance. Sensor (1) is set at lower position on the ground towards the entrance. In normal use of restroom, it will detect the distance from feet of user to the sensor when the user enters. Sensor (2) is set at a higher position, 50 centimeters above the ground. It is located towards the toilet. In normal use, it will fetch the distance change signal according to toilet using.

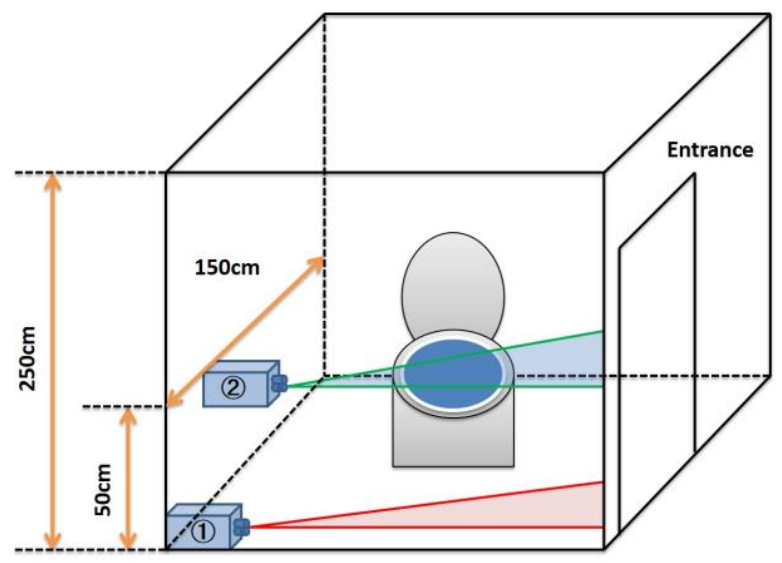

Fig. 3. Layout of experimental environment 
The experimental flow is designed as Fig. 4 shows. When target person enters the restroom, as Fig. 4 (a) shows, he/she will get into the detect range of sensor (1). The detected distance will change from initial value according to the user's movement towards the sensor. When the target person uses the toilet, he/she will move to the detect range of sensor (2), getting out from sensor (1) range as Fig. 4 (b) shows. Then, when the user exit the restroom, the he/she will get out from the range of sensor (1) to the one of sensor (2) as shown in Fig. 4 (c). If there is any accident, such as the user falls down on the ground, the detected signal will be completely different from the one of normal process.

\subsection{Experimental results and discussion}

According to the flow design, the experiments are implemented. There a male target person for exam the two groups of experiments, the normal use and use with falling

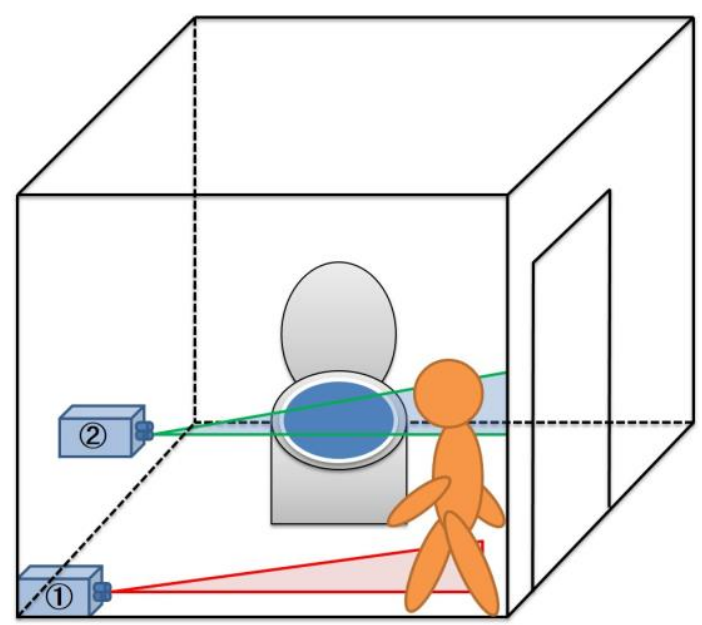

(a) Entrance

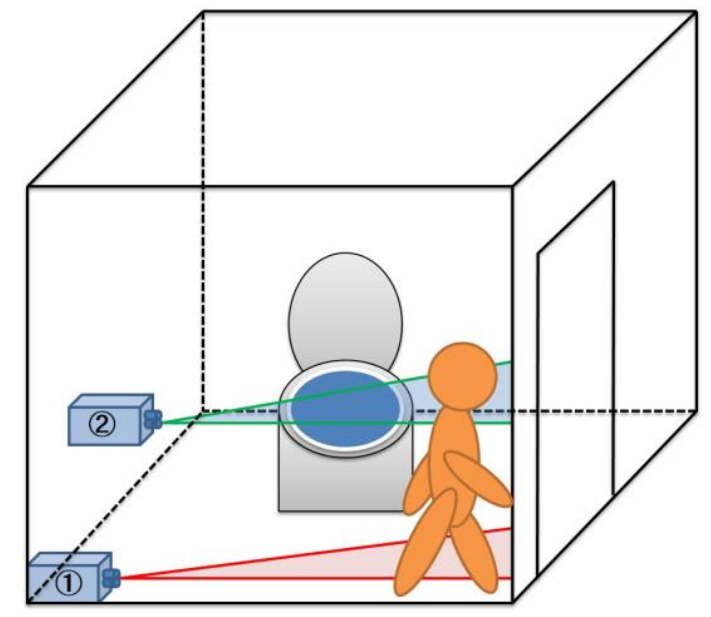

(c) Exit down. The target persons is in 20's with the heights of 176 $\mathrm{cm}$. The results of him are shown in Fig. 5 and Fig. 6. As we see in the figures, the horizontal axis is time and the vertical axis show distance from sensors. The solid line shows the signal of sensor (1), and the dashed line is the signal variation of sensor (2). The experiment time was set to 5 minus. Within the limited time, the target persons completed the motions according to the experimental design.

Figure 5 shows that the user get into the restroom in $20^{\text {th }}$ second around. The entering motion caused the detected distance varied from $200 \mathrm{~cm}$ to $50 \mathrm{~cm}$ within 5 second, and then returned to the initial value of $200 \mathrm{~cm}$. In normal use of toilet, the detected distance of sensor (2) varied from 200 $\mathrm{cm}$ to about $90 \mathrm{~cm}$. It returned to the initial value when the user exited the restroom. At that time, the detected distance of sensor (1) varied again to about $50 \mathrm{~cm}$ and went back to $200 \mathrm{~cm}$ within $5 \mathrm{~cm}$. The normal use can be recognized

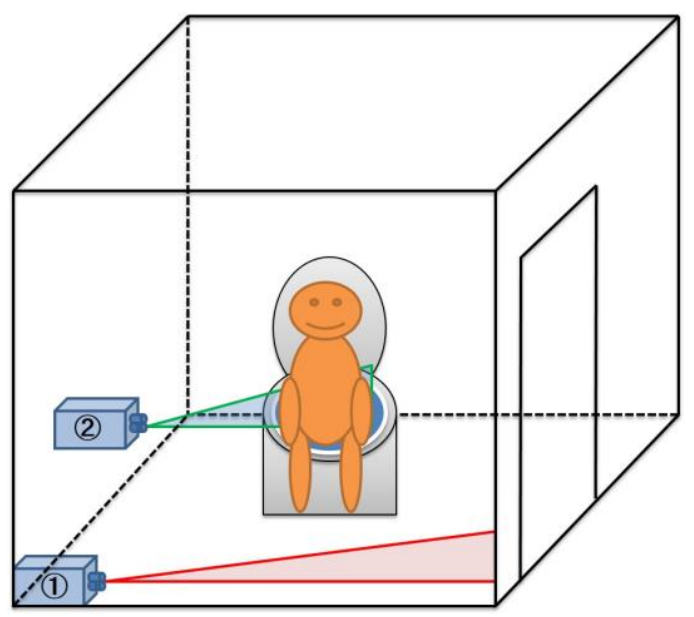

(b) In use

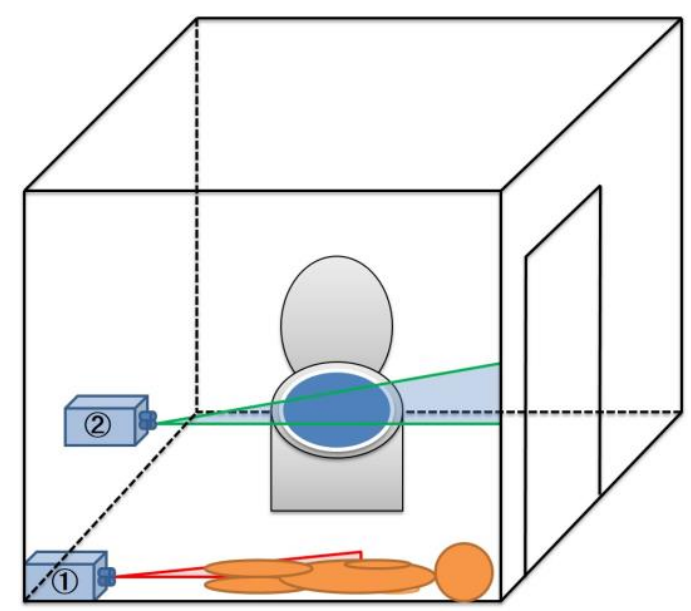

(d) Fall down

Fig. 4. The experimental setting 


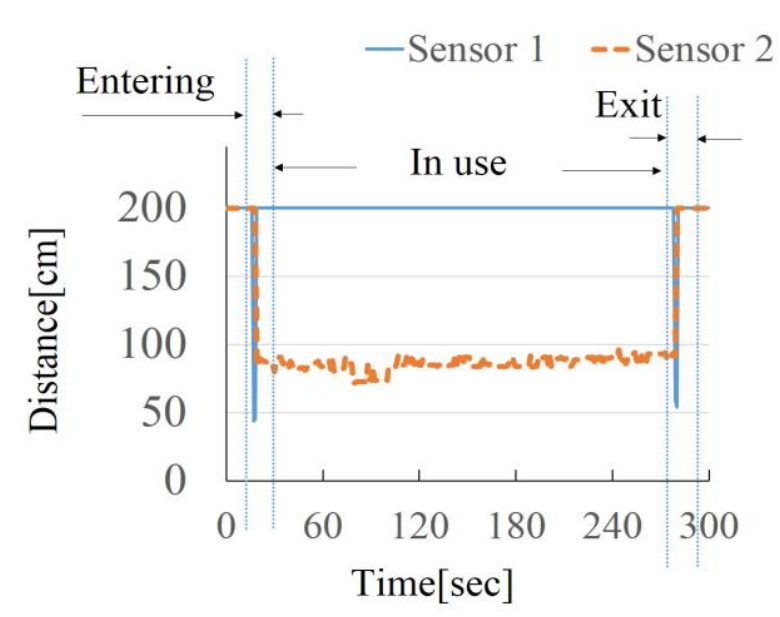

Fig. 5. Results of normal use

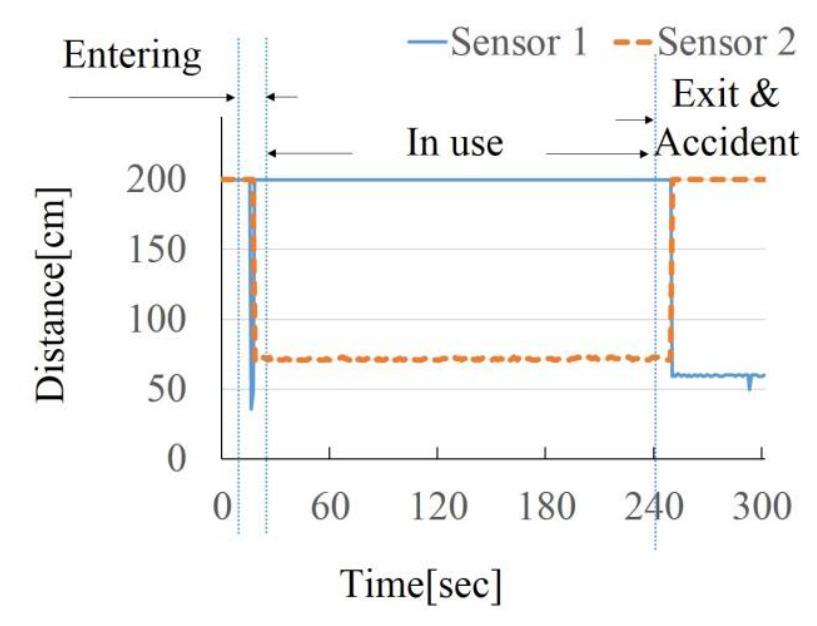

Fig. 7. Results of accident in exiting

clearly with the distance variations of the two sensors.

In detecting of accident situations, we considered the target person fell down when entered and exited the restroom. The results are shown in Fig. 6 and Fig. 7. In Fig. 6, the target person went into the rest room in the $20^{\text {th }}$ second around. The falling down happened when the user walked in. It is clear as we see in Fig. 6 that sensor (1) responded to the distance change of falling down. The signal from sensor (1) stayed around the distance at $50 \mathrm{~cm}$. The signal from sensor (2) did not change in the process. Figure 7 shows the situation of having accident when the target person goes out from the rest room. The signal from sensor (1) stayed around the distance at $50 \mathrm{~cm}$ after the falling down. The signal from sensor (2) went back to the initial value when the user fallen down. Comparing with the experimental results, it is clear that the unmoral use of restroom with falling down accident can be

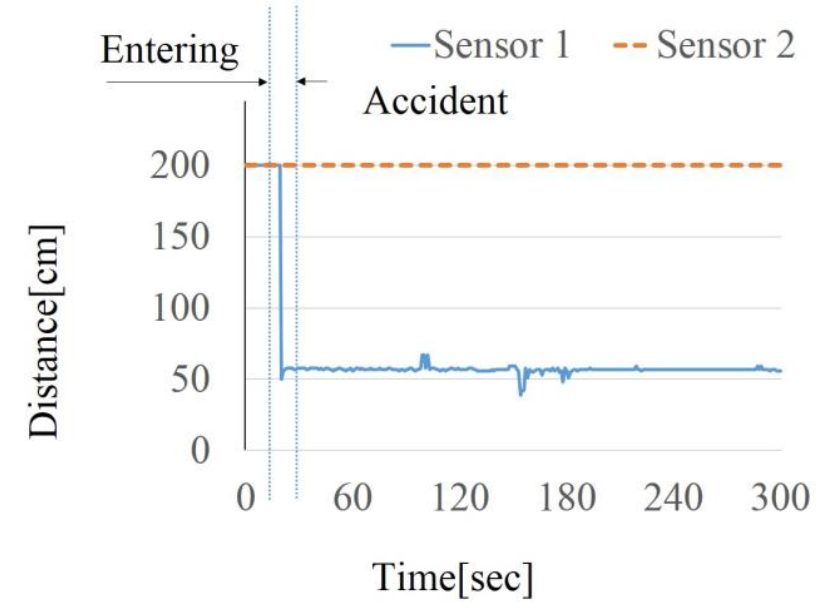

Fig. 6. Results of accident in entering

effectively detected.

\section{Conclusions}

The paper reports the simple application of ultrasonic sensor for safety confirmation in restroom. The proposed method was confirmed effective by experiments. The proposed method is supposed to be applied in the safety confirmation system for elders living along in outlying island. In following research, it will be employed together with further advanced sensors for human motion detection in more complex environment.

\section{Acknowledgment}

The work was supported by the founding of University Reform Action Plan, Center of Community (COC) in Hiroshima National College of Maritime Technology.

\section{References}

(1) K. Tanaka, K. Haruyama, Y. Yamada, Y. Wakasa and T. Akashi, Movement Confirmation System using Ultrasonic Radar and Power Line Communications for senior citizens, Japanese Journal of Applied IT Healthcare, Vol.2, No.2, pp. 95-103, 2008

(2) S. Nakashima, S. Mu, S. Okabe, K. Tanaka, Y. Wakasa, Y. Kitazono, and S. Serikawa, Restroom Human Detection Using One-Dimensional Brightness Distribution Sensor, Software Engineering SNPD, Studies in Computational Intelligence, Roger Lee, Ed., Springer, Vol. 492, pp. 1-10, July. 1, 2013 